\title{
FeliX - mit Algebra Geometrie machen
}

\author{
Prof. Dr. Reinhard Oldenburg \\ Institut für Didaktik der Mathematik und Informatik \\ Johann Wolfgang Goethe Universität Frankfurt a. M. \\ Senckenberganlage 9 und 11 \\ 60325 Frankfurt am Main
}

oldenbur@math.uni-frankfurt.de

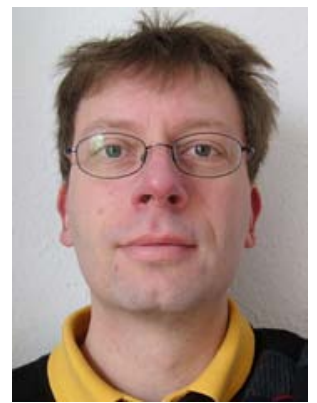

\section{Zusammenfassung}

Zeichnen oder rechnen? Viele Probleme kann man geometrisch oder algebraisch lösen. Die Übersetzung zwischen geometrischer und algebraischer Welt kann man mit dem Programm FeliX live erleben: Beide Sichtweisen sind gleichberechtigt integriert.

\section{Zum Einstieg: Eine Kurbel mit Gummiband}

Mathematik liefert die Sprache, mit der man viele Situationen beschreiben kann. In Abbildung 1 ist eine drehbare Kurbel $K$ gezeigt, an der ein Gummiband montiert ist, dessen zweites Ende an einem Punkt $F$ fixiert ist. Der Mittelpunkt $M$ des Bandes ist markiert. Wenn man jetzt kurbelt, auf welcher Bahn bewegt sich dann $M$ ? Vielleicht reicht die Vorstellungskraft aus, das Problem zu lösen. Im Normalfall kann man sich eine Zeichnung machen, eine Reihe von Mittelpunkten für verschiedene Kurbelstellungen einzeichnen und so die Bahn gewinnen. Alternativ rechnet man algebraisch: Wenn der Drehpunkt der Kurbel im Ursprung liegt, und der Kurbelarm die Radiuslänge $r$ hat, erfüllen die Koordinaten $\left(x_{K}, y_{K}\right)$ von $K$ die Kreisgleichung $x_{K}^{2}+y_{K}^{2}=r^{2}$. Der Mittelpunkt $M\left(x_{M}, y_{M}\right)$ von $K$ und $F\left(x_{F}, y_{F}\right)$ erfüllt die Gleichungen $2 x_{M}=x_{F}+x_{K}$ und $2 y_{M}=$ $y_{F}+y_{K}$. Wenn man diese Gleichungen nach $\left(x_{K}, y_{K}\right)$ auflöst und in die Kreisgleichung einsetzt, erhält man: $\left(2 x_{M}-x_{F}\right)^{2}+\left(2 y_{M}-y_{F}\right)^{2}=r^{2}$. Dies ist eine Kreisgleichung mit Mittelpunkt $\left(\frac{x_{F}}{2}, \frac{y_{F}}{2}\right)$, wie man besser erkennt, wenn man die ganze Gleichung durch $4=2^{2}$ dividiert: $\left(x_{M}-\frac{x_{F}}{2}\right)^{2}+\left(y_{M}-\frac{y_{F}}{2}\right)^{2}=\left(\frac{r}{2}\right)^{2}$.

Dass man eine Kreisbahn erhält, kann man auch geometrisch verstehen: Das Gummiband realisiert eine zentrische Streckung. Der Vorteil der (mühevollen) algebraischen Lösung ist, dass man mit dem Ergebnis gut weitere Fragen untersuchen kann, z. B. Schnittpunkte berechnen oder Tangenten anlegen. Vor allem aber kann man die algebraische Lösungsmethode systematisch auch auf kompliziertere Probleme anwenden, und dabei kann man sich von Computeralgebrasystemen helfen lassen.

Besonders elegant gestaltet sich die Lösung, wenn man FeliX verwendet. Dieses Geometrieprogramm basiert auf Computeralgebra und kann solche algebraischen Berechnungen durchführen. Abbildung 2 zeigt die entsprechende Lösung. Allerdings geht es bei FeliX nicht nur darum, Lösungen zu erhalten, sonderen vor allem darum, den Zusammenhang von Algebra und Geometrie besser zu verstehen.

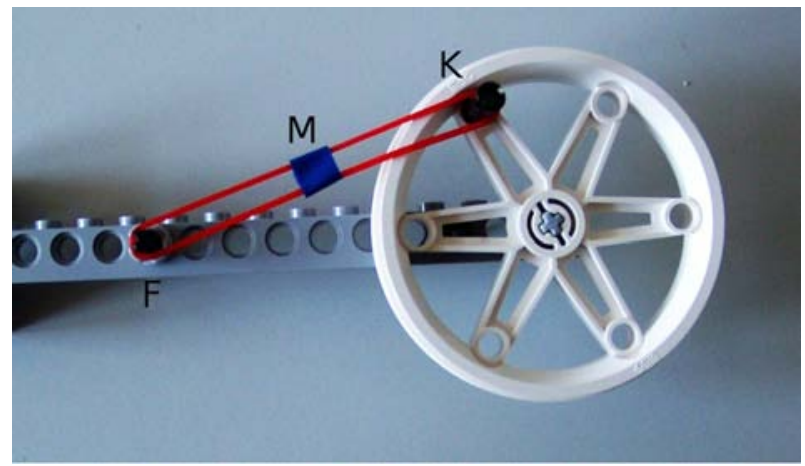

Abbildung 1: Eine Kurbel dehnt ein Gummiband 


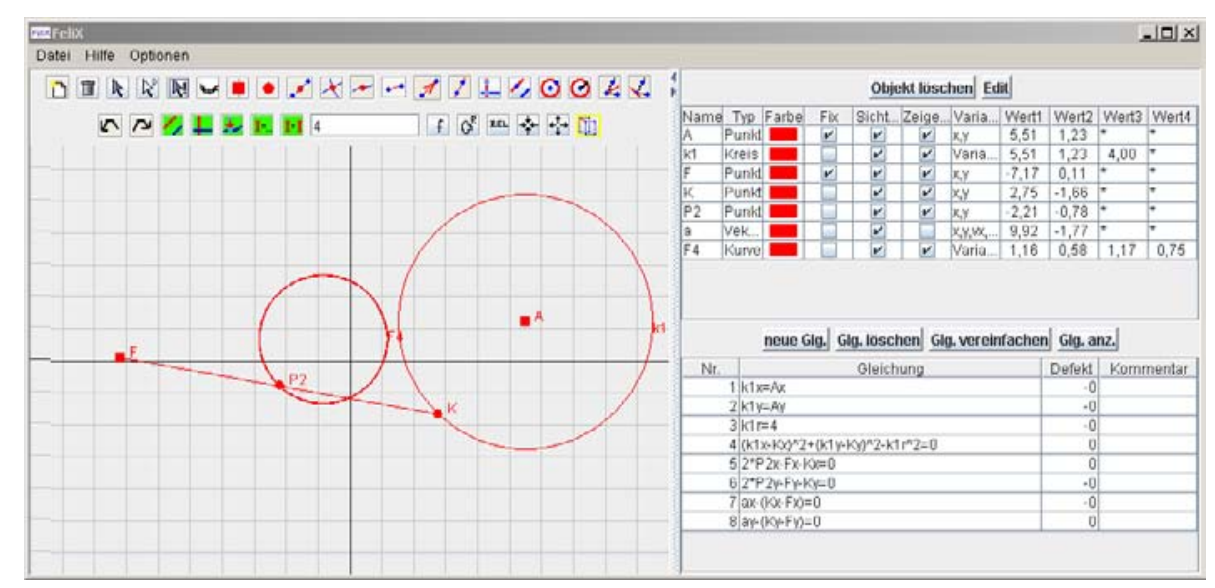

Abbildung 2: Die Situation aus Abbildung 1 mathematisch realisiert mit FeliX

\section{FeliX - ein Algebra-Geometrie-Programm}

Die Grundidee von FeliX ist recht einfach: Man erzeugt im Geometriefenster geometrische Objekte wie Punkte, Geraden, Strecken und Kreise, die mit der Maus verschoben werden können. In der Objektliste werden die Objekte mit ihren numerischen Koordinaten angezeigt. Dort kann man die Koordinaten auch ändern, was sofort eine Neupositionierung im Geometriefenster nach sich zieht.

\begin{tabular}{|l|l|l|}
\hline Objektart & Variablen & Bedeutung \\
\hline $\begin{array}{l}\text { Punkt / point } \\
\text { P }\end{array}$ & Px, Py & $\begin{array}{l}\text { Kartesische Koordi- } \\
\text { naten des Punktes }\end{array}$ \\
\hline $\begin{array}{l}\text { Kreis / circle } \\
\text { K }\end{array}$ & Kx, Ky, Kr & $\begin{array}{l}\text { Mittelpunktkoordinaten } \\
\text { und Radius }\end{array}$ \\
\hline $\begin{array}{l}\text { Grade / line } \\
\mathrm{L}\end{array}$ & La, Lb, Lc & $\begin{array}{l}\text { Koeffizienten in der } \\
\text { Gleichung ax+by=c }\end{array}$ \\
\hline $\begin{array}{l}\text { Strecke V } \\
\text { oder Vektor V }\end{array}$ & Vx, Vy & $\begin{array}{l}\text { Vektorkomponenten } \\
\text { Vx, Vy }\end{array}$ \\
\hline
\end{tabular}

Tabelle 1: Die Objektarten in FeliX und ihre Koordinaten

Objekte alleine sind aber noch nicht so spannend. Interessant wird die virtuelle geometrische Welt erst durch die Beziehungen zwischen den Objekten. Solche Beziehungen sind beispielsweise, dass ein Punkt Mittelpunkt zweier anderer ist, dass zwei Geraden parallel oder orthogonal sind, dass ein Punkt auf einer Geraden oder auf einem Kreis liegt oder der Schnittpunkt zweier Objekte ist. Solche Beziehungen lassen sich alle mit Gleichungen ausdrücken und die Idee von FeliX ist die, dass das System beim Bewegen von Objekten mit der Maus (oder durch direkte Eingabe von neuen Koordinaten) immer die Gültigkeit einer Reihe von Gleichungen einhält. Dabei ist es egal, ob die Gleichungen vom Benutzer von Hand in die Gleichungstabelle eingetragen worden sind, oder ob sie über die Konstruktionsbuttons wie „Mittelpunkt erzeugen“ erzeugt wurden.

Ein Beispiel: Der Benutzer erzeugt zwei Punkte $A$ und $B$, wählt danach das Mittelpunktswerkzeug aus der Buttonleiste und klickt die beiden Punkte an. Es erscheint im Geometriefenster ein neuer Punkt $M$ und in der Gleichungstabelle tauchen zwei neue Gleichungen auf: $2 \star M x=A x+B x$ und $2 \star M y=A y+B y$. Egal, an welchem Punkt man dann wie zieht, immer bleibt $M$ der Mittelpunkt von $A$ und $B$. Das ändert sich erst, wenn man die Gleichungen ändert, z.B. in $3 * M x=2 * A x+B x$ und $3 * M y=2 * A y+B y$. Haben Sie eine Vorstellung, was das bewirkt?

Weitere interessante Möglichkeiten ergeben sich daraus, dass man auch Ungleichungen eingeben kann. Damit kann man z.B. verhindern, dass Kreise sich überlappen. Daraus resultiert ein Verhalten, wie man es von physikalischen Scheiben erwartet: Die Kreise schieben sich gegenseitig weg.

Für das weitere ist noch die Unterscheidung von fixen und nicht fixen Objekten nötig: Fixe Punkte werden als kleine Quadrate, nicht fixe als kleine Kreisscheiben dargestellt. Das Verhalten der Konstruktion beim Ziehen wird durch die Zugregel bestimmt:

Fixe Objekte ändern ihre Koordinaten nur, wenn sie direkt bewegt werden, aber nie mittelbar in Reaktion auf die Bewegung anderer Punkte.

Ob ein Objekt fix ist oder nicht, kann jederzeit umgeschaltet werden.

\section{Kurven}

Konstruktionen wie die in Abschnitt 1 führen oft zu Punkten, die sich nur auf einer bestimmten Kurve bewegen lassen. Schöne Beispiele sind die Kegelschnitte. Eine Ellipse ist etwa die Menge aller Punkte $P$, für die die Summe der Abstände zu zwei gegebenen Punkten $A$ und $B$ eine bestimmte Konstante ist. Um eine Ellipse in FeliX zu erzeugen, kreiert man also zwei fixe Punkte $A$ und $B$ und einen nicht-fixen Punkt $P$. Die Abstände muss man mit dem Satz des Pythagoras berechnen (sqrt ist die Wurzelfunktion). Eine geeignete Gleichung ist: $\operatorname{sqrt}\left((\mathrm{Px}-\mathrm{Ax})^{\wedge} 2+(\mathrm{Py}-\mathrm{Ay})^{\wedge} 2\right)+$ $\operatorname{sqrt}\left((P x-B x)^{\wedge} 2+(P y-B y)^{\wedge} 2\right)=10$. Diese Gleichung bewirkt, dass sich $P$ mit der Maus nur noch auf der Ellipse ziehen lässt, für die alle Punkte die Abstandssumme 10 zu den beiden gegebenen Punkten haben. Um die Ellipse auch als Kurve zu sehen, benutzt man den Kurven-Button und klickt dann auf $P$. FeliX berechnet dann die Gleichung der Kurve mit den Koordinaten von $A$ und $B$ als variablen Parametern und 
zeichnet sie dann. Mit dieser Technik lassen sich viele interessante Kurven erzeugen, u. a. Herzkurven (Kardioiden) oder die „Hundelinie“, die eigentlich „Konchoide des Nikomedes" (siehe [1]) heißt. Hier soll aber lieber nochmal das Lego ausgepackt werden.

\section{Legoaffe}

Das Kurbelbeispiel aus Abschnitt 1 ist etwas künstlich: Wo sind schon Gummibänder gespannt und wer interessiert sich für deren Mittelpunkt? Etwas anders liegen die Dinge bei dem Lego-Seilbahn-Roboter in Abbildung 3. Es ist schon spannend ihm zuzusehen, wie er sich an einem gespannten Seil vorwärts hangelt: Die Hand wird schnell von hinten nach vorne geführt und dann zieht er sich ein Stück weiter. Aber ob sich die Hand dabei auf einem Kreis oder einer Ellipse oder einer anderen Kurve bewegt, kann man kaum beurteilen. Mit FeliX lassen sich die Kurbel und die Schubstange aber schnell nachbilden und die Bahnkurve berechnen.

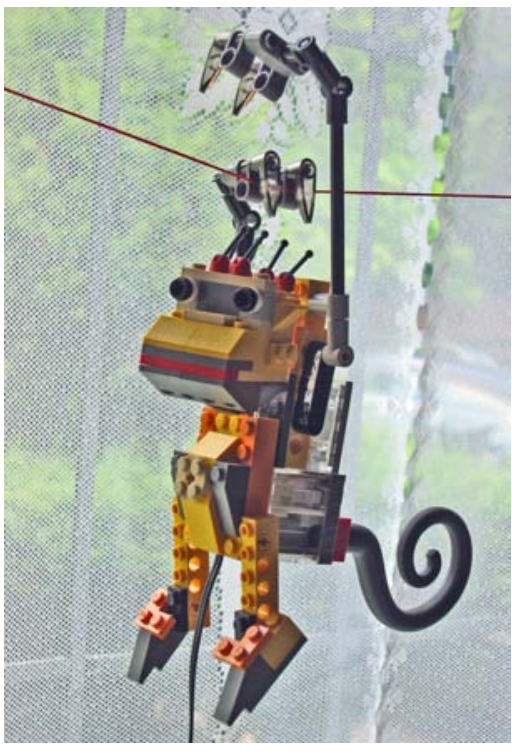

Abbildung 3: Der Motor-Kletteraffe von Lego

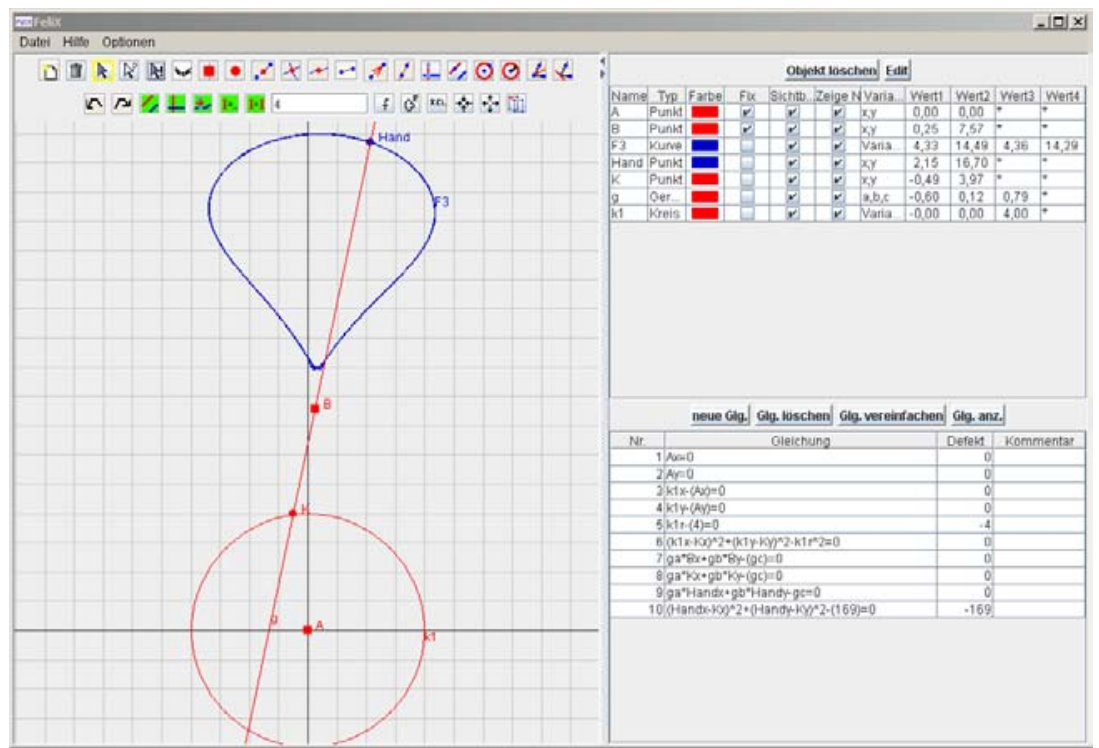

Abbildung 4: Der Kletteraffe in FeliX: Der Kreismittelpunkt ist die Motorachse, der Punkt auf dem Kreis der „Ellbogen“.

Man sieht, dass sich die Hand auf einer komplexen Bahn bewegt.

\section{Schluss}

FeliX existiert in verschiedenen Varianten: Neben der kleinen eindimensionalen Fassung FeliX1D, bei der man Variablen auf einem Zahlenstrahl verschiebt, gibt es zwei Ausgaben, mit denen man die hier beschriebenen zweidimensionalen Probleme lösen kann. Eine setzt auf dem kommerziellen Computeralgebrasystem $\mathrm{Mu}$ PAD auf, die andere benötigt MuPAD nicht und ist dadurch leichter zu installieren und vollständig kostenlos, wenn auch gegenwärtig nicht ganz so leistungsfähig. Das Programm kann von der Homepage des Autors ${ }^{5}$ kostenlos herunter geladen werden. Es setzt eine JavaLaufzeitumgebung der aktuellen Version 1.6 voraus, die ebenfalls frei erhältlich ist.

\section{Links und Literatur}

[1] D. Haftendorn, Konchoide des Nikomedes (Hundekurve), siehe www. fh-lueneburg.de/mathelehramt/mathe-lehramt.htm.

[2] R. Oldenburg, Bidirektionale Verknüpfung von Computeralgebra und dynamischer Geometrie, Journal für Mathematikdidaktik, 26 (2005), 249-273.

\footnotetext{
$5_{\text {www }}$ math.uni-frankfurt.de/ oldenbur/
} 